\title{
Article \\ Inflation from the Symmetry of the Generalized Cosmological Model
}

\author{
Koblandy Yerzhanov*(D, Gulnur Bauyrzhan (D), Aziza Altaibayeva (D) and Ratbay Myrzakulov (D) \\ Department of General and Theoretical Physics, L.N. Gumilyov Eurasian National University, \\ Nur-Sultan Z01C0X1, Kazakhstan; 880914401677@enu.kz (G.B.); altaibayeva_ab_1@enu.kz (A.A.); \\ 540201300113@enu.kz (R.M.) \\ * Correspondence: yerzhanov_kk@enu.kz
}

Citation: Yerzhanov, K.; Bauyrzhan G.; Altaibayeva, A.; Myrzakulov, R. Inflation from the Symmetry of the Generalized Cosmological Model. Symmetry 2021, 13, 2254. https:// doi.org/10.3390/sym13122254

Academic Editors: Roberto Passante and Iver H. Brevik

Received: 27 September 2021

Accepted: 16 November 2021

Published: 26 November 2021

Publisher's Note: MDPI stays neutral with regard to jurisdictional claims in published maps and institutional affiliations.

\begin{abstract}
It is shown that the inflationary model is the result of the symmetry of the generalized $F(R, T, X, \varphi)$-cosmological model using the Noether symmetry. It leads to a solution, a particular case of which is Starobinsky's cosmological model. It is shown that even in the more particular case of cosmological models $F(R, X, \varphi)$ and $F(T, X, \varphi)$ the Monge-Ampère equation is still obtained, one of the solutions including the Starobinsky model. For these models, it is shown that one can obtain both power-law and exponential solutions for the scale factor from the Euler-Lagrange equations. In this case, the scalar field $\varphi$ has similar time dependences, exponential and exponential. The resulting form of the Lagrangian of the model allows us to consider it as a model with $R^{2}$ or $X^{2}$. However, it is also shown that previously less studied models with a non-minimal relationship between $R$ and $X$ are important, as one of the possible models. It is shown that in this case the power-law model can have a limited evolutionary period with a negative value of the kinetic term.
\end{abstract}

Keywords: symmetry; inflation; alternative gravity; cosmology; torsion; scalar

\section{Introduction}

Constantly appearing new cosmological data, on the one hand, call attention to the generalized theory of gravity. However, on the other hand, this leads to the need to clarify the general theory of relativity. It should help determine the causes of the accelerated expansion of the universe in the initial period and in our time SNe Ia [1,2]. The current accelerated expansion of the Universe can be described by different models. The simplest of these is the model of general relativity with the cosmological constant $\Lambda$. This model is currently referred to as the $\Lambda C D M$ standard cosmological model. This model can describe the current state of the universe. However, there is a need to include in the description the initial inflationary period of the development of the Universe. A more complex version of the description is the inclusion of various fields in the cosmological model such as scalar field $\phi$. Here, to generalize the influence of the scalar field on the cosmological model, we use the k-essence model with the Lagrangian density in generalized form $L=F(\phi, X)[3,4]$ based on the k-inflation model [5]. We do not consider other forms of fields here, like tachyon field, phantom (ghost) field, dilatonic dark energy, Chaplygin gas, or generalisation of scalar and fermion fields g-essence [6-8].

The next way, used to describe the observed inflationary phenomena, is $F(R)$-gravity. As a special case, it includes a model of gravity with a square member of the Ricci scalar $R^{2}$, which can be seen as a generalization of general relativity, which describes the Starobinsky inflation. As an alternative to this model, a teleparallel gravity model is being developed, which in generalized form can be represented as $F(T)$ [9], where $T$ is the torsion scalar $[10,11]$.

As a generalization of these two ways of describing the universe, the model of the $F(R, T)$ modified theory-Myrzakulov gravity $[12,13]$ was used. Here, the Lagrangian is a function of two variables, such as the Ricci scalar and torsion scalar [14-16]. In this paper, 
we will try to consider the most generalized model, which would include in an arbitrary form $F(R, T, X, \phi)$ both Myrzakulov's gravity and a scalar field in the form of k-essence.

To consider this model, the Noether symmetry is used here. $m$ is the matter part of the action To study the model, we use the Noether symmetry. This approach is widely used in various branches of physics, including cosmology. Here, we consider the FriedmannLemaitre-Robertson-Walker (FRW) metric with zero curvature.

To simplify the consideration, we will consider a model without the matter part of the action, since the purpose of the article is to consider the generalized model by symmetry methods, for which the influence of the material part is not significant. In addition, for simplicity, consider the model in the framework of the Friedman-Lemaitre-RobertsonWalker (FRW) metric with zero curvature.

\section{2. $F(R, T, X, \phi)$ Gravity}

Consider a general manifold with curvature and torsion, for which a standard torsionfree Riemannian manifold will be a part of it. In this manifold, we define the metric $g_{\mu \nu}$ and the metric-compatible path connection $\Gamma$ so that the scalar curvature $R$ and the torsion scalar $T$ correspond to the dynamical connection $\Gamma$. In general, this relationship can be written as [17-19]

$$
\Gamma_{i j}^{h}=L_{i j}^{h}-K_{i j}^{h}+N_{i j}^{h},
$$

where $L_{i j}{ }^{h}$ is the Levi-Civita connection associated with the given metric $g, K_{i j}{ }^{h}$ denotes the contorsion tensor and $N_{i j}{ }^{h}$ is the disformation tensor. Here,

$$
N^{\alpha}{ }_{\mu \nu}=\frac{1}{2} g^{\alpha \beta}\left(Q_{\beta \mu \nu}-Q_{\mu \beta \nu}-Q_{\nu \beta \mu}\right)
$$

where

$$
Q_{\alpha \mu \nu}=\nabla_{\alpha} g_{\mu \nu}
$$

and

$$
Q^{\alpha \mu v}=-g^{\alpha \beta} \nabla_{\beta} g^{\mu \nu}
$$

Here, we assume that

$$
Q_{\alpha \mu \nu}=\nabla_{\alpha} g_{\mu \nu}=0
$$

Here, we define the torsion tensor as

$$
T_{i j}^{h}=\Gamma_{i j}^{h}-\Gamma_{j i}^{h} .
$$

Then, in terms of the torsion tensor, the contorsion tensor expressed as

$$
K_{i j}^{h}=\frac{1}{2}\left(-T_{i j}{ }^{h}+T_{j}{ }_{i}-T_{i j}^{h}\right) \text {, }
$$

where the contorsion tensor with the antisymmetry property $K_{i}{ }^{j h}=-K_{i}{ }^{h j}$. The Ricci curvature of the dynamical connection has the form

$$
R_{i j}=\tilde{R}_{i j}+\tilde{\nabla}_{j} K_{h i}{ }^{h}-\tilde{\nabla}_{h} K_{j i}{ }^{h}+K_{j i}{ }^{p} K_{h p}{ }^{h}-K_{h i}{ }^{p} K_{j p}{ }^{h} .
$$

Hence, we get the scalar curvature as

$$
R=R_{l}+u,
$$


where the function $u$ has the form

$$
u=\tilde{\nabla}_{j} K_{h}{ }^{j h}-\tilde{\nabla}_{h} K_{j}{ }^{j h}+K_{j}^{j p} K_{h p}{ }^{h}-K_{h}{ }^{j p} K_{j p}{ }^{h} .
$$

Here, $\tilde{R}_{i j}$ and $R_{l}$ are the Ricci and the scalar curvature of the Levi-Civita connection induced by the metric $g_{\mu v}$. Similarly to the curvature scalar $R$, now we introduce the following invariant that is the torsion scalar as

$$
T=S^{\rho \mu v} T_{\rho \mu \nu},
$$

where

$$
S^{\rho \mu \nu}=0.5\left(K^{\mu \nu \rho}-g^{\rho v} T_{\sigma}^{\sigma \mu}+g^{\rho \mu} T_{\sigma}^{\sigma v}\right) .
$$

As the curvature scalar $R$, we now can decompose the expression for the torsion scalar as

$$
T=T_{w}+v,
$$

where $v$ is some real function to be defined as the other function $u$. We are now ready to write the action of this gravity that is given by [10-12]

$$
S=\frac{1}{2 k^{2}} \int d^{4} x \sqrt{-g} F(R, T)+S_{m}
$$

where

$$
\begin{aligned}
S_{m} & =\int d^{4} x \sqrt{-g} L_{m}, \\
R & =R_{l}+u, \\
T & =T_{w}+v .
\end{aligned}
$$

Here, $k^{2}=8 \pi G, g$ and $L_{m}$ are metric determinant and the matter Lagrangian, respectively, and $F(R, T)$ is an arbitrary function of curvature scalar $R$ and torsion scalar $T, R_{w}$ is the curvature scalar for the Weitzenböck spacetime, $T_{r}$ is the torsion scalar for the Riemann spacetime, $R_{l}$ is the curvature scalar of the Riemann spacetime, and $T_{w}$ is the torsion scalar of the Weitzenböck spacetime. In the above,

$$
\begin{aligned}
& u=u\left(x^{j}, g_{i j}, \frac{\partial g_{i j}}{\partial x^{k}}, \ldots\right), \\
& v=v\left(x^{j}, g_{i j}, \frac{\partial g_{i j}}{\partial x^{k}}, \ldots\right),
\end{aligned}
$$

are some real functions with the necessary properties and their arguments, $(i, j, k=$ $0,1,2,3)$. We define $|e| \equiv \operatorname{det}\left(e_{\mu}^{i}\right)=\sqrt{-g}$ in order to connect the two formalisms. It is to verify that, from the MG, both $F(T)$ and $F(R)$ gravities can be immediately recovered. Variation of the action gives the following field equations [13]

$$
\begin{aligned}
& \frac{1}{4} e_{A}^{\mu} f(R, T)+e^{-1}\left(e e_{A}^{\sigma} S_{\sigma}^{\mu v}\right) \frac{\partial f(R, T)}{\partial T} \partial_{v}+e_{A v}\left[\left(\nabla^{\mu} \nabla^{v}-g^{\mu \nu} \nabla^{\lambda} \nabla_{\lambda}\right) \frac{\partial f(R, T)}{\partial R}-\frac{\partial f(R, T)}{\partial R} R^{\mu v}\right] \\
& +e_{A}^{\sigma} S_{\sigma}^{\mu v}\left(\frac{\partial^{2} f(R, T)}{\partial T^{2}} \partial_{v} T+\frac{\partial^{2} f(R, T)}{\partial T \partial R} \partial_{v} R\right)-\frac{\partial f(R, T)}{\partial T} e_{A}^{\gamma} S^{\rho \beta \mu} T_{\rho \beta \gamma}=4 \pi G e_{A}^{v} T^{(m)} v^{\mu} .
\end{aligned}
$$

Traditionally cosmological models are considered within the FRW metric. Consider it similar to the interval $d s^{2}=d t^{2}-a(t)^{2}\left(d x^{2}+d y^{2}+d z^{2}\right)$. Let us start with the model in its most general form as $F(R, T, X, \phi)$ gravity, the action for which has the following form:

$$
S=2 \pi^{2} \int d t a^{3}\left\{F-\lambda_{1}\left[R-u-6\left(\frac{\ddot{a}}{a}+\frac{\dot{a}^{2}}{a^{2}}\right)\right]-\lambda_{2}\left[T-v+6\left(\frac{\dot{a}^{2}}{a^{2}}\right)\right]-\lambda_{3}\left[X-\frac{1}{2} \dot{\phi}^{2}\right]\right\} .
$$


Here, $a$-scale factor, $u, v$-some functions $u=u\left(\Gamma_{\mu v}^{\rho} ; x_{i} ; g_{i j}, \dot{g}_{i j}, \ddot{g_{i j}}, \ldots ; f_{j}\right), \quad v=$ $v\left(\Gamma^{\rho}{ }_{\mu v} ; x_{i} ; g_{i j}, \dot{g}_{i j}, \ddot{g_{i j}}, \ldots ; g_{j}\right)$, but, for this paper, we will consider them as functions of $t, \dot{t}, \ddot{t}$.

As is well known, we can write the Euler-Lagrange equations for FRW model as

$$
\frac{d}{d t} \frac{\partial L}{\partial \dot{a}}=\frac{\partial L}{\partial a} \quad \frac{d}{d t} \frac{\partial L}{\partial \dot{R}}=\frac{\partial L}{\partial R} \quad \frac{d}{d t} \frac{\partial L}{\partial \dot{T}}=\frac{\partial L}{\partial T} \quad \frac{d}{d t} \frac{\partial L}{\partial \dot{X}}=\frac{\partial L}{\partial X} \quad \frac{d}{d t} \frac{\partial L}{\partial \dot{\phi}}=\frac{\partial L}{\partial \phi}
$$

with the energy condition

$$
E_{L}=\frac{\partial L}{\partial \dot{a}} \dot{a}+\frac{\partial L}{\partial \dot{R}} \dot{R}+\frac{\partial L}{\partial \dot{T}} \dot{T}+\frac{\partial L}{\partial \dot{X}} \dot{X}+\frac{\partial L}{\partial \dot{\phi}} \dot{\phi}-L=0 .
$$

Here,

$$
\begin{aligned}
& R=u+R^{(L C)}, \\
& T=v+T^{(W C)} .
\end{aligned}
$$

Here, $R^{(L C)}$ is the curvature scalar corresponding to the Levi-Civita connection with the vanishing torsion, and $T^{(W)}$ is the torsion scalar for the purely Weitzenböck connection with the vanishing curvature.

For FRW, we can write it as

$$
\begin{aligned}
R & =u+6\left(\dot{H}+2 H^{2}\right), \\
T & =v-6 H^{2}, \\
X & =\frac{1}{2} \dot{\phi}^{2} .
\end{aligned}
$$

From the Euler-Lagrange equations for $F(R, T, X, \phi)$ by $R, T$, and $X$, we obtain

$$
\lambda_{1}=F_{R}, \quad \lambda_{2}=F_{T}, \quad \lambda_{3}=F_{X} .
$$

Here, and after denoting $F(R, T, X, \phi)$ as $F$, and $F_{R}, F_{T}, F_{X}$ are derivations of the $F$ function by $R, T$, and $X$, respectively. Integrate by parts, and, if we consider that $u, v$ depends linearly on $a, \dot{a}, \ddot{a}$ and $R, T$, respectively, then we can rewrite the Lagrangian as

$$
\begin{aligned}
L= & a^{3}\left[F-b_{1}(R-u) F_{R}-b_{2}(T-v) F_{T}\right]-6 a \dot{a}^{2}\left[b_{1} F_{R}+b_{2} F_{T}\right] \\
& -6 a^{2} \dot{a}\left[\dot{R} F_{R R}+\dot{T} F_{R T}+\dot{X} F_{R X}+\dot{\phi} F_{R \phi}\right]-a^{3} F_{X}\left[X-\frac{1}{2} \dot{\phi}^{2}\right] .
\end{aligned}
$$

Here, $b_{1}, b_{2}$ are some constants. A similar result is obtained for $u(a, \dot{a})$ and $v(a, \dot{a})$. Thus, there is not a big difference between $u, v$ which linearly depends on $R, T$ or only $a, \dot{a}$. Furthermore, we can use point-like Lagrangian, where $u, v$ are some functions of $a, \dot{a}$ only as

$$
\begin{aligned}
L= & a^{3}\left[F-(R-u) F_{R}-(T-v) F_{T}\right]-6 a \dot{a}^{2}\left[F_{R}+F_{T}\right]-6 a^{2} \dot{a}\left[\dot{R} F_{R R}\right. \\
& \left.+\dot{T} F_{R T}+\dot{X} F_{R X}+\dot{\phi} F_{R \phi}\right]-a^{3} F_{X}\left[X-\frac{1}{2} \dot{\phi}^{2}\right] .
\end{aligned}
$$

For this most general form of the cosmological model with a scalar field, the EulerLagrange equations will have the following very complex form:

$$
F-A F_{R}-B F_{T}-\left(u_{\dot{a}} \frac{a}{3}-4 H\right) \dot{F_{R}}-\left(v_{\dot{a}} \frac{a}{3}-4 H\right) \dot{F_{T}}-2 \ddot{F_{R}}-F_{X}\left(X-\frac{1}{2} \dot{\phi}^{2}\right)=0
$$

where

$$
\begin{aligned}
A & =R-u-\frac{a}{3} u_{a}+u_{\dot{a}} \dot{a}+u_{\dot{a} a} \dot{a} \frac{a}{3}+u_{\dot{a} \dot{a}} \ddot{a} \frac{a}{3}-4 \dot{H}+6 H^{2} \\
B & =T-v-\frac{a}{3} v_{a}+v_{\dot{a}} \dot{a}+v_{\dot{a} a} \dot{a} \frac{a}{3}+v_{\dot{a} \dot{a}} \ddot{a} \frac{a}{3}-4 \dot{H}-6 H^{2} .
\end{aligned}
$$


Klein-Gordon equation here is

$$
F_{\phi}-3 H F_{X} \dot{\phi}-\left[\dot{R} F_{X R}+\dot{T} F_{X T}+\dot{X} F_{X X}+\dot{\phi} F_{X \phi}\right] \dot{\phi}-F_{X} \ddot{\phi}=0 .
$$

The energy condition [10] gives

$$
\begin{aligned}
E_{L}= & a^{3}\left[F_{X}\left(X+\frac{1}{2} \dot{\phi}^{2}\right)-F+F_{R}\left(\dot{a} u_{\dot{a}}+6 H^{2}+R-u\right)+F_{T}\left(\dot{a} v_{\dot{a}}-6 H^{2}+T-v\right)\right. \\
& \left.+6 H\left(\dot{R} F_{R R}+\dot{T} F_{R T}+\dot{X} F_{R X}+\dot{\phi} F_{R \varphi}\right)\right]=0 .
\end{aligned}
$$

As is evident from these equations, it is very difficult to solve them in their most general form. To solve it, it is important to obtain an analytical solution to the Lagrangian of the model.

\section{The Noether Symmetries Approach}

The use of symmetry methods has a long history in physics. It provides conservation laws. Thus, the Standard Model is built on local gauge symmetry. The Yang-Mills theory used to construct the Standard Model, in particular, uses the Slavnov-Taylor-WardTakahashi identities. Here, we use the analogue of the Ward-Takahashi identity is the Noether symmetry. In other words, we can say that conservation laws are manifestations of Noether's theorem. By this theorem, for a dynamical system with coordinates, which is described by the Lagrangian with the system of Euler-Lagrange equations, there exists a vector field $X$ for which the derivative of the Lagrangian with respect to $X$ must vanish. Noether symmetries are often used in cosmology for scalar-tensor theory-for example, one of the first [20]. Thus, from the point of view of applying Noether symmetry to models with a scalar field, our work is not original. The novelty here lies only in the application of the Noether symmetry to the generalized model of gravity, which gives new results. The choice of this symmetry is widely used in alternative theories of gravity and cosmology [21]. The shape of the Lagrangian can be found from the Noether symmetry condition [22-25]:

$$
X L=0,
$$

where

$$
X=\alpha \frac{\partial}{\partial a}+\beta \frac{\partial}{\partial R}+\gamma \frac{\partial}{\partial T}+\delta \frac{\partial}{\partial X}+\epsilon \frac{\partial}{\partial \varphi}+\dot{\alpha} \frac{\partial}{\partial \dot{a}}+\dot{\beta} \frac{\partial}{\partial \dot{R}}+\dot{\gamma} \frac{\partial}{\partial \dot{T}}+\dot{\delta} \frac{\partial}{\partial \dot{X}}+\dot{\epsilon} \frac{\partial}{\partial \dot{\varphi}} .
$$

Here, the functions $\alpha, \beta, \gamma, \delta, \epsilon$ depend on the variables $a, R, T, X, \varphi$.

By this, we have:

For $F(R, X, \varphi)$

$$
\begin{array}{r}
6\left(\alpha F_{R}+\beta a F_{R R}+\delta a F_{R X}+\epsilon a F_{R \varphi}+2 \alpha_{a} a F_{R}+\beta_{a} a^{2} F_{R R}+\delta_{a} a^{2} F_{R X}+\epsilon_{a} a^{2} F_{R \varphi}\right) \dot{a}^{2} \\
+\dot{\varphi}^{2}\left(\epsilon_{\varphi} a^{3} F_{X}-6 \alpha_{\varphi} a^{2} F_{R \varphi}+\frac{3}{2} \alpha a^{2} F_{X}+\frac{1}{2} \beta a^{3} F_{X R}+\frac{1}{2} \delta a^{3} F_{X X}+\frac{1}{2} \epsilon a^{3} F_{X \varphi}\right) \\
+6 a \dot{a} \dot{R}\left(\beta a F_{R R R}+\delta a F_{R R X}+\epsilon a F_{R R \varphi}+2 \alpha_{R} F_{R}+\left(\alpha_{a} a+\beta_{R} a+2 \alpha\right) F_{R R}+\delta_{R} a F_{R X}+\epsilon_{R} a F_{R \varphi}\right) \\
+6 a \dot{a} \dot{X}\left(2 \alpha_{X} F_{R}+a \beta_{X} F_{R R}+\beta a F_{R X R}+\delta a F_{R X X}+\epsilon a F_{R X \varphi}+\left(2 \alpha+\alpha_{a} a+\delta_{X} a\right) F_{R X}+\epsilon_{X} a F_{R \varphi}\right) \\
+6 a \dot{a} \dot{\varphi}\left(a \beta_{\varphi} F_{R R}+2 \alpha F_{R \varphi}+\beta a F_{R \varphi R}+\delta a F_{R \varphi X}+\epsilon a F_{R \varphi \varphi}+\alpha_{a} a F_{R \varphi}+\delta_{\varphi} a F_{R X}+\epsilon_{\varphi} a F_{R \varphi}\right) \\
+12 a \dot{a} \dot{\varphi} \alpha_{\varphi} F_{R}+6 \dot{R}^{2} \alpha_{R} a^{2} F_{R R}+6 \dot{X}^{2} \alpha_{X} a^{2} F_{R X}-a^{3} \dot{a} \dot{\varphi} \epsilon_{a} F_{X}+6 a^{2} \dot{R} \dot{X}\left(\alpha_{X} F_{R R}+\alpha_{R} F_{R X}\right) \\
+\dot{R} \dot{\varphi} a^{2}\left(6 \alpha_{\varphi} F_{R R}-\epsilon_{R} a F_{X}+6 \alpha_{R} F_{R \varphi}\right)+\dot{X} \dot{\varphi}\left(-\epsilon_{X} a^{3} F_{X}+6 \alpha_{X} a^{2} F_{R \varphi}+6 \alpha_{\varphi} a^{2} F_{R X}\right) \\
+3 a^{2} \alpha\left[F-(R-u) F_{R}+\frac{1}{3} a u_{a} F_{R}\right]-\beta a^{3}(R-u) F_{R R}-\delta a^{3}(R-u) F_{R X} \\
+a^{3} u_{\dot{a}} F_{R}\left(\dot{a} \alpha_{a}+\dot{R} \alpha_{R}+\dot{X} \alpha_{X}+\dot{\varphi} \alpha_{\varphi}\right)-\left(\alpha 3 F_{X}+\beta a F_{X R}+\delta a F_{X X}+\epsilon a F_{X \varphi}\right) X a^{2} \\
+\epsilon a^{3}\left[F_{\varphi}-(R-u) F_{R \varphi}\right]=0 .
\end{array}
$$

In addition, as a system of equations: 


$$
\begin{aligned}
\dot{a}^{2}: \quad & \alpha F_{R}+\beta a F_{R R}+\delta a F_{R X}+\epsilon a F_{R \varphi}+2 \alpha_{a} a F_{R} \\
& +\beta_{a} a^{2} F_{R R}+\delta_{a} a^{2} F_{R X}+\epsilon_{a} a^{2} F_{R \varphi}=0, \\
\dot{R}^{2}: \quad & 6 \alpha_{R} a^{2} F_{R R}=0, \\
\dot{X}^{2}: \quad & 6 \alpha_{X} a^{2} F_{R X}=0, \\
\dot{\varphi}^{2}: \quad & \epsilon_{\varphi} a^{3} F_{X}-6 \alpha_{\varphi} a^{2} F_{R \varphi}+\frac{3}{2} \alpha a^{2} F_{X}+\frac{1}{2} \beta a^{3} F_{X R}+\frac{1}{2} \delta a^{3} F_{X X} \\
& +\frac{1}{2} \epsilon a^{3} F_{X \varphi}=0, \\
& 2 \alpha F_{R R}+\beta a F_{R R R}+\delta a F_{R R X}+\epsilon a F_{R R \varphi} \\
\dot{a} \dot{R}: \quad & +2 \alpha_{R} F_{R}+\alpha_{a} a F_{R R}+\beta_{R} a F_{R R}+\delta_{R} a F_{R X}+\epsilon_{R} a F_{R \varphi}=0, \\
\dot{a} \dot{X}: \quad & 2 \alpha_{X} F_{R}+a \beta_{X} F_{R R}+2 \alpha F_{R X}+\beta a F_{R X} \\
& +\delta a F_{R X X}+\epsilon a F_{R X}+\alpha_{a} a F_{R X}+\delta_{X} a F_{R X}+\epsilon_{X} a F_{R \varphi}=0, \\
\dot{a} \dot{\varphi}: \quad & 2 \alpha_{\varphi} F_{R}+a \beta_{\varphi} F_{R R}-\epsilon_{a} \frac{a^{2}}{6} F_{X}+2 \alpha F_{R \varphi} \\
& +\beta a F_{R \varphi R}+\delta a F_{R \varphi X}+\epsilon a F_{R \varphi \varphi}+\alpha_{a} a F_{R \varphi}+\delta_{\varphi} a F_{R X}+\epsilon_{\varphi} a F_{R \varphi}=0, \\
\dot{R} \dot{X}: \quad & \alpha_{X} a^{2} F_{R R}+\alpha_{R} a^{2} F_{R X}=0, \\
\dot{R} \dot{\varphi}: \quad & 6 \alpha_{\varphi} a^{2} F_{R R}-\epsilon_{R} a^{3} F_{X}+6 \alpha_{R} a^{2} F_{R \varphi}=0, \\
\dot{X} \dot{\varphi}: \quad & -\epsilon_{X} a^{3} F_{X}+6 \alpha_{X} a^{2} F_{R \varphi}+6 \alpha_{\varphi} a^{2} F_{R X}=0, \\
& 3 \alpha\left[F-(R-u) F_{R}+\frac{1}{3} a u_{a} F_{R}\right]-\beta a(R-u) F_{R R}-\delta a(R-u) F_{R X} \\
& +\epsilon a\left[F_{\varphi}-(R-u) F_{R \varphi}\right]+\dot{a} \alpha_{a} a u_{\dot{a}} F_{R}+\dot{\varphi} \alpha_{\varphi} a a u_{\dot{a}} F_{R}+\dot{R} \alpha_{R} a u_{\dot{a}} F_{R} \\
& +\dot{X} \alpha_{X} a u_{\dot{a}} F_{R}-\left(\alpha 3 F_{X}+\beta a F_{X R}+\delta a F_{X X}+\epsilon a F_{X \varphi}\right) X=0 .
\end{aligned}
$$

For $F(R, T, X, \varphi)$

$$
\begin{aligned}
& 6 \dot{a}^{2}\left(\alpha\left[F_{R}-F_{T}\right]+\beta a\left[F_{R R}-F_{T R}\right]+\gamma a\left[F_{R T}-F_{T T}\right]+\delta a\left[F_{R X}-F_{T X}\right]+\epsilon a\left[F_{R \varphi}-F_{T \varphi}\right]\right) \\
& +6 \dot{a}^{2}\left(\alpha_{a} 2 a\left[F_{R}-F_{T}\right]+\beta_{a} a^{2} F_{R R}+\gamma_{a} a^{2} F_{T R}+\delta_{a} a^{2} F_{R X}+\epsilon_{a} a^{2} F_{R \varphi}\right)+\dot{R}^{2} 6 \alpha_{R} a^{2} F_{R R} \\
& +\dot{T}^{2} 6 \alpha_{T} a^{2} F_{R T}+\dot{X}^{2} 6 \alpha_{X} a^{2} F_{R X} \\
& +\dot{\varphi}^{2}\left(\epsilon_{\varphi} a^{3} F_{X}-6 \alpha_{\varphi} a^{2} F_{R \varphi}+\alpha \frac{3}{2} a^{2} F_{X}+\beta \frac{1}{2} a^{3} F_{X R}+\gamma \frac{1}{2} a^{3} F_{X T}+\delta \frac{1}{2} a^{3} F_{X X}+\epsilon \frac{1}{2} a^{3} F_{X \varphi}\right) \\
& +\dot{a} \dot{R} 6 a\left(\beta a F_{R R R}+\gamma a F_{R R T}+\delta a F_{R R X}+\epsilon a F_{R R \varphi}+\gamma_{R} a F_{T R}+\delta_{R} a F_{R X}+\epsilon_{R} a F_{R \varphi}\right) \\
& +\dot{a} \dot{R} 6 a\left(2 \alpha_{R}\left[F_{R}-F_{T}\right]+\left(\alpha_{a} a+\beta_{R} a+2 \alpha\right) F_{R R}\right) \\
& +\dot{a} \operatorname{T} 6 a\left(\beta a F_{T R R}+\gamma a F_{T R T}+\delta a F_{T R X}+\epsilon a F_{T R \varphi}+2 \alpha_{T}\left[F_{R}-F_{T}\right]\right) \\
& +\dot{a} T 6 a\left(\left(\alpha_{a} a+2 \alpha+\gamma_{T} a\right) F_{T R}+\beta_{T} a F_{R R}+\delta_{T} a F_{R X}+\epsilon_{T} a F_{R \varphi}\right) \\
& +\dot{a} \dot{X} 6 a\left(2 \alpha_{X}\left[F_{R}-F_{T}\right]+a \beta_{X} F_{R R}+a \gamma_{X} F_{T R}+\beta a F_{R X R}+\gamma a F_{R X T}+\delta a F_{R X X}+\epsilon a F_{R X \varphi}\right) \\
& +\dot{a} \dot{X} 6 a\left(\left(2 \alpha+\alpha_{a} a+\delta_{X} a\right) F_{R X}+\epsilon_{X} a F_{R \varphi}\right) \\
& +\dot{a} \dot{\varphi} 6 a\left(2 \alpha_{\varphi}\left[F_{R}-F_{T}\right]+a \beta_{\varphi} F_{R R}+a \gamma_{\varphi} F_{T R}+\epsilon_{a} \frac{a^{2}}{6} F_{X}+2 \alpha F_{R \varphi}\right) \\
& +\dot{a} \dot{\varphi} 6 a\left(\beta a F_{R \varphi R}+\gamma a F_{R \varphi T}+\delta a F_{R \varphi X}+\epsilon a F_{R \varphi \varphi}+\alpha_{a} a F_{R \varphi}+\delta_{\varphi} a F_{R X}+\epsilon_{\varphi} a F_{R \varphi}\right) \\
& +\dot{R} \dot{T} 6 a^{2}\left(\alpha_{R} F_{T R}+\alpha_{T} F_{R R}\right)+\dot{R} \dot{X} 6 a^{2}\left(\alpha_{X} F_{R R}+\alpha_{R} F_{R X}\right) \\
& +6 \dot{T} \dot{X} a^{2}\left(\alpha_{X} F_{T R}+\alpha_{T} F_{R X}\right)+\dot{R} \dot{\varphi} a^{2}\left(6 \alpha_{\varphi} F_{R R}+\epsilon_{R} a F_{X}+6 \alpha_{R} F_{R \varphi}\right) \\
& +\dot{T} \dot{\varphi}\left(6 \alpha_{\varphi} a^{2} F_{T R}+\epsilon_{T} a^{3} F_{X}+6 \alpha_{T} a^{2} F_{R \varphi}\right)+\dot{X} \dot{\varphi}\left(\epsilon_{X} a^{3} F_{X}+6 \alpha_{X} a^{2} F_{R \varphi}+6 \alpha_{\varphi} a^{2} F_{R X}\right) \\
& +3 a^{2} \alpha\left[F-(R-u) F_{R}-(T-v) F_{T}+\frac{1}{3} a\left(u_{a} F_{R}+v_{a} F_{T}\right)\right] \\
& +\beta a^{3}\left[-(R-u) F_{R R}-(T-v) F_{T R}\right]+\gamma a^{3}\left[-(R-u) F_{R T}-(T-v) F_{T T}\right] \\
& +\delta a^{3}\left[-(R-u) F_{R X}-(T-v) F_{T X}\right]+\epsilon a^{3}\left[F_{\varphi}-(R-u) F_{R \varphi}-(T-v) F_{T \varphi}\right] \\
& +a^{3}\left[u_{\dot{a}} F_{R}+v_{\dot{a}} F_{T}\right]\left(\dot{a} \alpha_{a}+\dot{R} \alpha_{R}+\dot{T} \alpha_{T}+\dot{X} \alpha_{X}+\dot{\varphi} \alpha_{\varphi}\right) \\
& -\left(\alpha 3 F_{X}+\beta a F_{X R}+\gamma a F_{X T}+\delta a F_{X X}+\epsilon a F_{X \varphi}\right) X a^{2}=0 .
\end{aligned}
$$


In addition, as a system of equations:

$$
\begin{aligned}
& \dot{a}^{2}: \quad \alpha\left[F_{R}+F_{T}\right]+\beta a\left[F_{R R}+F_{T R}\right]+\gamma a\left[F_{R T}+F_{T T}\right] \\
& +\delta a\left[F_{R X}+F_{T X}\right]+\epsilon a\left[F_{R \varphi}+F_{T \varphi}\right]+\alpha_{a} 2 a\left[F_{R}+F_{T}\right] \\
& +\beta_{a} a^{2} F_{R R}+\gamma_{a} a^{2} F_{T R}+\delta_{a} a^{2} F_{R X}+\epsilon_{a} a^{2} F_{R \varphi}=0, \\
& \dot{R}^{2}: \quad 6 \alpha_{R} a^{2} F_{R R}=0, \\
& \dot{T}^{2}: \quad 6 \alpha_{T} a^{2} F_{R T}=0 \text {, } \\
& \dot{X}^{2}: \quad 6 \alpha_{X} a^{2} F_{R X}=0 \text {, } \\
& \dot{\varphi}^{2}: \quad a^{3}\left(\epsilon_{\varphi} F_{X}-6 \frac{\alpha_{\varphi}}{a} F_{R \varphi}+\frac{3 \alpha}{2 a} F_{X}+\frac{\beta}{2} F_{X R}+\frac{\gamma}{2} F_{X T}+\frac{\delta}{2} F_{X X}+\frac{\epsilon}{2} F_{X \varphi}\right)=0, \\
& \dot{a} \dot{R}: \quad 2 \alpha F_{R R}+\beta a F_{R R R}+\gamma a F_{R R T}+\delta a F_{R R X}+\epsilon a F_{R R \varphi} \\
& +2 \alpha_{R}\left[F_{R}+F_{T}\right]+\alpha_{a} a F_{R R}+\beta_{R} a F_{R R}+\gamma_{R} a F_{T R}+\delta_{R} a F_{R X}+\epsilon_{R} a F_{R \varphi}=0, \\
& \dot{a} \dot{T}: \quad 2 \alpha F_{T R}+\beta a F_{T R R}+\gamma a F_{T R T}+\delta a F_{T R X}+\epsilon a F_{T R \varphi} \\
& +2 \alpha_{T}\left[F_{R}+F_{T}\right]+\alpha_{a} a F_{T R}+\beta_{T} a F_{R R}+\gamma_{T} a F_{T R}+\delta_{T} a F_{R X}+\epsilon_{T} a F_{R \varphi}=0, \\
& \dot{a} \dot{X}: \quad 2 \alpha_{X}\left[F_{R}+F_{T}\right]+a \beta_{X} F_{R R}+a \gamma_{X} F_{T R}+2 \alpha F_{R X}+\beta a F_{R X R} \\
& +\gamma a F_{R X T}+\delta a F_{R X X}+\epsilon a F_{R X \varphi}+\alpha_{a} a F_{R X}+\delta_{X} a F_{R X}+\epsilon_{X} a F_{R \varphi}=0, \\
& \dot{a} \dot{\varphi}: \quad 2 \alpha_{\varphi}\left[F_{R}+F_{T}\right]+a \beta_{\varphi} F_{R R}+a \gamma_{\varphi} F_{T R}-\epsilon_{a} \frac{a^{2}}{6} F_{X}+2 \alpha F_{R \varphi} \\
& +\beta a F_{R \varphi R}+\gamma a F_{R \varphi T}+\delta a F_{R \varphi X}+\epsilon a F_{R \varphi \varphi}+\alpha_{a} a F_{R \varphi} \\
& +\delta_{\varphi} a F_{R X}+\epsilon_{\varphi} a F_{R \varphi}=0 \text {, } \\
& \dot{R} \dot{T}: \quad \alpha_{R} a^{2} F_{T R}+\alpha_{T} a^{2} F_{R R}=0, \\
& \dot{R} \dot{X}: \quad \alpha_{X} a^{2} F_{R R}+\alpha_{R} a^{2} F_{R X}=0 \text {, } \\
& \dot{T} \dot{X}: \quad \alpha_{X} a^{2} F_{T R}+\alpha_{T} a^{2} F_{R X}=0 \text {, } \\
& \dot{R} \dot{\varphi}: \quad 6 \alpha_{\varphi} a^{2} F_{R R}-\epsilon_{R} a^{3} F_{X}+6 \alpha_{R} a^{2} F_{R \varphi}=0, \\
& \dot{T} \dot{\varphi}: \quad 6 \alpha_{\varphi} a^{2} F_{T R}-\epsilon_{T} a^{3} F_{X}+6 \alpha_{T} a^{2} F_{R \varphi}=0, \\
& \dot{X} \dot{\varphi}: \quad-\epsilon_{X} a^{3} F_{X}+6 \alpha_{X} a^{2} F_{R \varphi}+6 \alpha_{\varphi} a^{2} F_{R X}=0, \\
& 3 \alpha\left[F-(R-u) F_{R}-(T-v) F_{T}+\frac{1}{3} a\left(u_{a} F_{R}+v_{a} F_{T}\right)\right] \\
& +\beta a\left[-(R-u) F_{R R}-(T-v) F_{T R}\right]+\gamma a\left[-(R-u) F_{R T}-(T-v) F_{T T}\right] \\
& +\delta a\left[-(R-u) F_{R X}-(T-v) F_{T X}\right]+\epsilon a\left[F_{\varphi}-(R-u) F_{R \varphi}-(T-v) F_{T \varphi}\right] \\
& +\dot{a} \alpha_{a} a\left[u_{\dot{a}} F_{R}+v_{\dot{a}} F_{T}\right]+\dot{\varphi} \alpha_{\varphi} a\left[u_{\dot{a}} F_{R}+v_{\dot{a}} F_{T}\right] \\
& +\dot{R} \alpha_{R} a\left[u_{\dot{a}} F_{R}+v_{\dot{a}} F_{T}\right]+\dot{T} \alpha_{T} a\left[u_{\dot{a}} F_{R}+v_{\dot{a}} F_{T}\right]+\dot{X} \alpha_{X} a\left[u_{\dot{a}} F_{R}+v_{\dot{a}} F_{T}\right] \\
& -\left(\alpha 3 F_{X}+\beta a F_{X R}+\gamma a F_{X T}+\delta a F_{X X}+\epsilon a F_{X \varphi}\right) X=0 .
\end{aligned}
$$

\section{The Noether Symmetries Solution}

The solution for the cases $F(R, T, X, \varphi)$ and $F(R, X, \varphi)$ looks the same. Thus, from a system of equations from equations for $\dot{R}^{2}, \dot{T}^{2}$ and $\dot{X}^{2}$, we have two solutions. The first solution is a linear equation $F=s_{1}(\varphi) R+s_{2}(\varphi) T+s_{3}(\varphi) X+s_{4}(\varphi)$ from $F_{R R}=F_{R T}=$ $F_{R X}=0$. In addition, for the second variant for a nonlinear solution, we have found that $\alpha_{R}=\alpha_{T}=\alpha_{X}=0$. From equations for $\dot{R} \dot{\varphi}, \dot{T} \dot{\varphi}$ and $\dot{X} \dot{\varphi}$, we have the next system of equations:

$$
\frac{\epsilon_{R}}{F_{R R}}=\frac{\epsilon_{T}}{F_{R T}}=\frac{\epsilon_{X}}{F_{R X}}=-\frac{6 \alpha_{\varphi}}{a F_{X}} .
$$


The last system of equations can be transformed to another form for $F(R, T, X, \varphi)$ as

$$
\begin{aligned}
& F_{R R} F_{X T}=F_{R T} F_{R X}, \\
& F_{R T} F_{X X}=F_{R X} F_{X T}, \\
& F_{R R} F_{X X}=F_{R X}^{2} .
\end{aligned}
$$

The last equation here is a homogeneous Monge-Ampere equation, but without dependence on $T$. Now, combine equations for $\dot{a} \dot{R}, \dot{a} \dot{T}$ and $\dot{a} \dot{X}$ as

$$
Z(a, \varphi)_{R}=Z(a, \varphi)_{T}=Z(a, \varphi)_{X}=0,
$$

where $Z(a, \varphi)=\left(2 \alpha+\alpha_{a} a\right) F_{R}+\beta a F_{R R}+\gamma a F_{R T}+\delta a F_{R X}+\epsilon a F_{R \varphi}$. This substitution is very useful because it allows for excluding functions $\beta, \gamma$, and $\delta$ from calculations. Now, it is possible with $\dot{a}^{2}$ to obtain the additional Monge-Ampere equation $F_{R R} F_{T T}=F_{R T}^{2}$. Solutions of Monge-Ampere equations involving one arbitrary function give the next result:

$$
F=f\left(C_{1}(\varphi) R+C_{2}(\varphi) T+C_{3}(\varphi) X, \varphi\right)+C_{4}(\varphi) R+C_{5}(\varphi) T+C_{6}(\varphi) X+C_{7}(\varphi),
$$

where $C_{1}, C_{2}, C_{3}, C_{4}, C_{5}, C_{6}, C_{7}$ are the functions of $\varphi$. Another solution is a solution involving arbitrary constants

$$
F=\left(C_{1}(\varphi) R+C_{2}(\varphi) T+C_{3}(\varphi) X\right)^{2}+C_{4}(\varphi) R+C_{5}(\varphi) T+C_{6}(\varphi) X+C_{7}(\varphi) .
$$

This solution gives us the same results as recent observations about the early time inflation well known as the Starobinsky model. The solution for the model $F(R, X, \varphi)$ is obtained similarly in a simpler way. Here, the Monge-Ampere equation is immediately obtained. In addition, since there are fewer model components, this equation is sufficient to solve from the next equation

$$
F_{R R} F_{X X}=F_{R X}^{2} .
$$

In this case, the solution involving arbitrary constants looks like

$$
F=\left(C_{8}(\varphi) R+C_{9}(\varphi) X\right)^{2}+C_{10}(\varphi) R+C_{11}(\varphi) X+C_{12}(\varphi),
$$

where $C_{8}, C_{9}, C_{10}, C_{11}, C_{12}$ are functions of $\varphi$.

\section{Conclusions}

In the resulting form, the Lagrangian is convenient and can be used to solve the above Euler Lagrange equations. However, the general solution is rather complicated. A special case can be found for the exponential scale factor for $F(R, T, X, \varphi)$, where, for simplicity, we take that $u=v=0$ and $a=a_{0} e^{H_{0} t}$, where $a_{0}, H_{0}$ are constants. For this case, if we accept that $C_{1}, C_{2}, \ldots, C_{6}$ - constants, then $\varphi$ will have an exponential solution

$$
\varphi=\varphi_{0} e^{t}
$$

Another special case is the power-law scale factor $a=a_{0} t^{n}$, where $n, a_{0}$ are constants with the same conditions as before. This scale factor will be a solution to the Euler-Lagrange equations if the scalar field also has a power-law dependence on time

$$
\varphi=\varphi_{0} t^{m}
$$

where $m, \varphi_{0}$ are constants. Solutions similar to those considered by us were widely studied earlier for special cases of k-essence and, for example, $F(R, X, \phi)$ cosmology (the last was considered for the model with linear $X$, which does not give the Monge-Ampere equation) [26]. Here, we have shown that these models that are power-law and exponential models can be obtained for a more general form of solutions. Such solutions can find 
application in models with $\mathrm{f}$ loop quantum cosmology and others [27-32]. Similar results can be found for the $F(R, X, \phi)$. It will be important to note here that the $\mathrm{n}$ in the power-law solution will depend only on $C_{8}, C_{9}, C_{1} 0$, and $\varphi_{0}$. Here, for the power-law solution, the parameter of the equation of state $\omega$ tends to -1 . Such models have already been discussed earlier, including models with $R^{2}$ and $X^{2}$ components. In fact, the $F(R, X, \varphi)$ model is reduced to these models, with the exception of the $R X$ component, which we can both zero out by obtaining the Starobinsky model at $C_{9}=0$, or $X^{2}$ at $C_{8}=0$. In the presence of the $\mathrm{XR}$ component, in this case, the behavior of $\mathrm{X}$ changes, which behaves as in the Figure 1. A feature of the solution will be the existence of a solution when $C_{8}$ and $C_{9}$ are not equal to zero. If we take the power-law solution for the scale factor here, we obtain a complex nonlinear differential inhomogeneous equation for the kinetic term $X$. Numerical analysis shows at some point in time that the value of $X$ in this model can be negative from the initial period of time to some point, which in general depends on the values of the functions $C$. Of course, such a decision cannot be considered completely correct, since, in this case, we considered only a power-law solution without an exponential one, which of course will prevail in the initial period of the evolution of the Universe. On the other hand, it should be noted that we used only a part of the Monge-Ampere solution. In the general case, it includes both a solution with a free function and possibly other solutions that can also affect the form of the scale factor and the kinetic term of the scalar field.

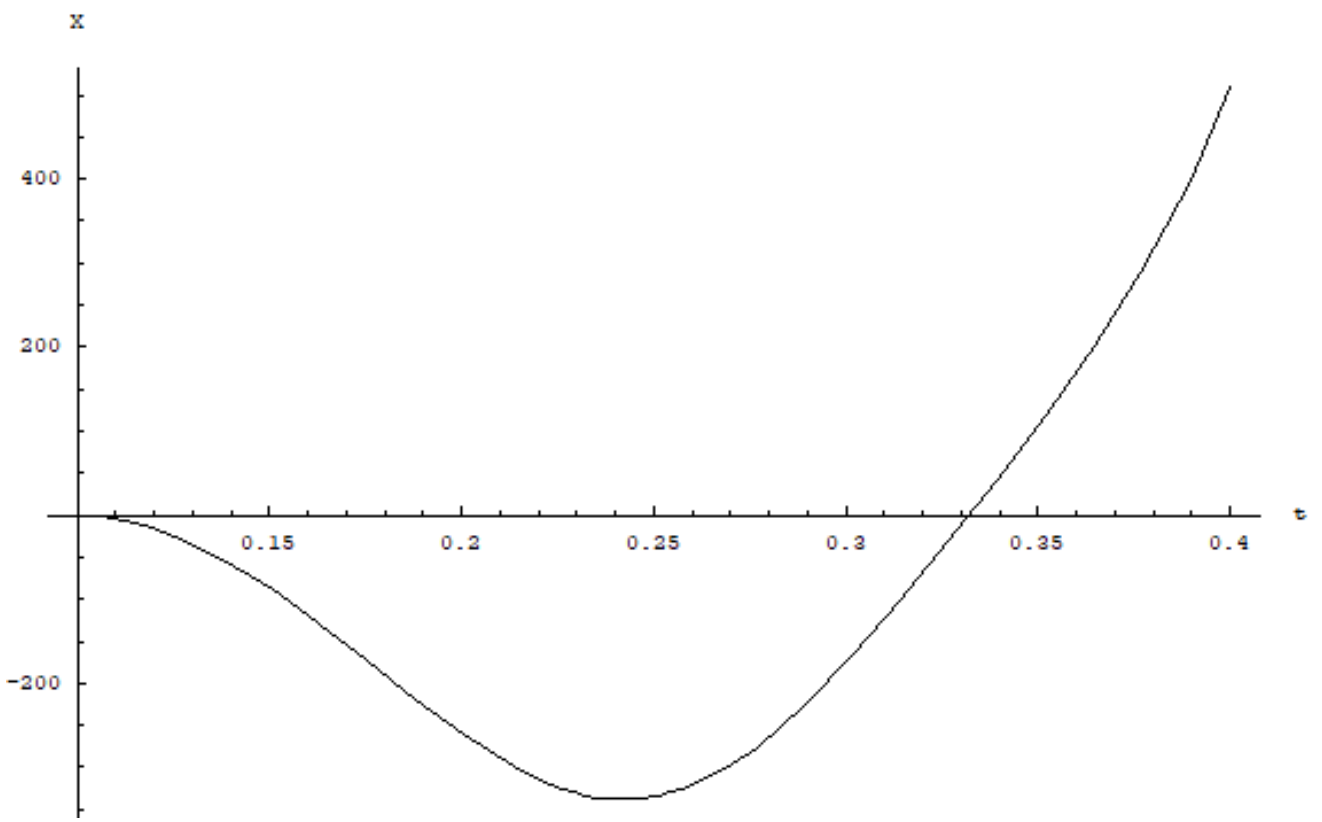

Figure 1. Qualitative demonstration of the dependence of the kinetic term $X$ on time $t$ for a power-law scale factor $a$ for a Lagrangian with a non-minimal dependence of $X$ on $R$.

In any case, here we were able to show that, when considering a generalized model with a scalar field, including $F(R)$ and $F(T)$-gravity, both together or separately, when using the Noether symmetry method, one can obtain the Starobinsky solution. It is also important that Starobinsky's solution is obtained even if we consider separately only $F(R)$ or only $F(T)$-gravity. That is, we will not be able to refute the necessity of $F(T)$-gravity here. In general, the solution for the Lagrangian can include more complex solutions. That is, include not only $R^{2}$, but also any other kinds of functions, such as exponential and/or high-order power functions, which can also be a source of inflation. In other words, it is shown here that, if the cosmological model includes the k-essence, the inflationary model of the Universe is a necessary consequence of the presence of symmetry. Unfortunately, since we took the model in its most general form, we do not have the opportunity to refine it more precisely in order to confirm the influence of symmetry on the evolution of the Universe through observations. A good opportunity for confirmation would be to detect 
the influence of the bound component of the $R X$ Lagrangian. However, in fact, this will not be a sufficient limitation of the model, since there is a possibility that the constants $C_{9}$ or $C_{3}$ are equal to zero. The model is then reduced to a family of well-designed k-essence models. Thus, the only way to confirm this mechanism of inflation of the Universe is to confirm the existence of the kinetic term of k-essence or non-minimally coupled kinetic term $X$ with $R$.

Author Contributions: Investigation, K.Y., A.A., G.B., and R.M.; Writing original draft: K.Y. and G.B.; Writing-review and editing, A.A. and R.M.; Supervision, R.M. All authors have read and agreed to the published version of the manuscript.

Funding: The work was financially supported by the Ministry of Education and Science of the Republic of Kazakhstan, Grant AP08957287.

Institutional Review Board Statement: Not applicable.

Informed Consent Statement: Not applicable.

Data Availability Statement: Not applicable.

Acknowledgments: We would like to thank the editors and the anonymous reviewers for their valuable comments and suggestions.

Conflicts of Interest: The authors declare no conflict of interest.

\section{References}

1. Perlmutter, S.; Aldering, G.; Goldhaber, G.; Knop, R.A.; Nugent, P.; Castro, P.G.; Deustua, S.; Fabbro, S.; Goobar, A.; Groom, D.E.; et al. Measurements of $\Omega$ and $\Lambda$ from 42 High-Redshift Supernovae. Astroph. J. 1999, 517, 565-586. [CrossRef]

2. Riess, A.G.; Filippenko, A.V.; Challis, P.; Clocchiattia, A.; Diercks, A.; Garnavich, P.M.; Gilliland, R.L.; Hogan, C.J.; Jha, S.; Kirshner, R.P.; et al. Observational Evidence from Supernovae for an Accelerating Universe and a Cosmological Constant. Astron. J. 1988, 116, 1009. [CrossRef]

3. Copeland, E.; Sami, M.; Tsujikawa, S. Dynamics of dark energy. Int. J. Mod. Phys. D 2006, 15, 1753. [CrossRef]

4. De Putter, R.; Linder, E.V. Kinetic k-essence and Quintessence. Astropart. Phys. 2007, 28, 263-272. [CrossRef]

5. Armendariz-Picon, C.; Damour, T.; Mukhanov, V. k-Inflation. Phys. Lett. B 1999, 458, 209-218. [CrossRef]

6. Myrzakulov, R.; Yerzhanov, K.; Yesmakhanova, K.; Tsyba, P.; Myrzakulov, N.; Nugmanova, G. g-Essence as the cosmic speed-up. Astrophys. Space Sci. 2012, 341, 681-688.

7. Bamba, K.; Myrzakulov, R.; Razina, O.; Yerzhanov, K. Cosmological evolution of equation of state for dark energy in G-essence models. Int. J. Mod. Phys. D 2013, 22, 1350023. [CrossRef]

8. Tsyba, P.Y.; Razina, O.V.; Suikimbayeva, N. Analysis cosmological tachyon and fermion model and observation data constraints. Int. J. Mod. Phys. D 2021, 2150114. [CrossRef]

9. Myrzakulov, R. Accelerating universe from F(T) gravity. Eur. Phys. J. C 2011, 71, 1752. [CrossRef]

10. Myrzakulov, R. Dark Energy in $F(R, T)$ Gravity. arXiv 2012, arXiv:1205.5266

11. Myrzakulov, R. FRW Cosmology in $F(R, T)$ gravity. Eur. Phys. J. C 2012, 72, 2203. [CrossRef]

12. Sharif, M.; Rani, S.; Myrzakulov, R. Analysis of F (R, T) Gravity Models Through Energy Con-ditions. Eur. Phys. J. Plus 2013, 128, 123. [CrossRef]

13. Pasqua, A.; Chattopadhyay, S.; Myrzakulov, R. A dark energy with higher order derivatives of $H$ in the modified gravity $f(R, T)$. ISRN High Energy Phys. 2014, 2014, 535010. [CrossRef]

14. Capozziello, S.; De Laurentis, M.; Myrzakulov, R. Noether Symmetry Approach for teleparallel-curvature cosmology. Int. J. Geom. Meth. Mod. Phys. 2015, 12, 1550095. [CrossRef]

15. Myrzakulov, R. F(T) gravity and k-essence. Gen. Relativ. Gravit. 2012, 44, 3059-3080. [CrossRef]

16. Gudekli, E.; Myrzakulov, N.; Yerzhanov, K.; Myrzakulov, R. Trace-anomaly driven inflation in $f(T)$ gravity with a cosmological constant. Astrophys. Space Sci. 2015, 357, 45. [CrossRef]

17. Gakis, V.; Krssak, M.; Said, J.L.; Saridakis, E.N. Conformal Gravity and Transformations in the Symmetric Teleparallel Framework. 2020, Phys. Rev. D 2020, 101, 064024. [CrossRef]

18. Fotios, A.K.; Spyros, B.; Saridakis, E.N. Observational constraints on Myrzakulov gravity. Phys. Rev. D 2020, $103,104013$.

19. Saridakis, E.N.; Myrzakul, S.N.; Myrzakulov, K.; Yerzhanov, K. Cosmological applications of F(R,T) gravity with dynamical curvature and torsion. Phys. Rev. D 2020, 102, 023525 [CrossRef]

20. Capozziello, S.; de Ritis, R.; Rubano, C.; Scudellaro, P. Noether symmetries in cosmology. Riv. Nuovo Cimento 1996, 19, 1. [CrossRef]

21. Massaeli, E.; Motaharfar, M.; Sepangi, H.R. General scalar-tensor cosmology: Analytical solu-tions via noether symmetry. Eur. Phys. J. C 2017, 77, 124. [CrossRef]

22. Sahlu, S.; Ntahompagaze, J.; Abebe, A.; de la Cruz-Dombriz, Á.; Mota, D.F. Scalar perturba-tions in $\mathrm{f}(\mathrm{T})$ gravity using the $1+3$ covariant approach. Eur. Phys. J. C 2020, 80, 422. [CrossRef] 
23. Darabi, F.; Asgharinya, S. f(R) scalar-tensor cosmology by Noether symmetry. Chin. J. Phys. 2015, 53, 040101.

24. Fu, G.Z.; Xing, C.C.; Na, W. The Scattering of Dirac Spinors in Rotating Spheroids. Eur. Phys. J. C 2020, 80, 582. [CrossRef]

25. Gao, Z.F.; Song, D.L.; Li, X.D.; Shan, H.; Wang, N. The equilibrium equations of Bos-on-Fermi systems in the Newtonian approximation. Astron. Nachr. 2019, 340, 241-246 [CrossRef]

26. Keskin, A.I.; Sirnak, U. Inflation and dark energy in $f(R, X, \phi)$ gravity. Mod. Phys. Lett. A 2018, 33, 1850215. [CrossRef]

27. Shahalam, M.; Sharma, M.; Wu, Q.; Wang, A. Pre-inflationary dynamics in loop quantum cosmology: Power-law potentials. Phys. Rev. D 2017, 96, 123533. [CrossRef]

28. Shahalam, M.; Sami, M.; Wang, A. Preinflationary dynamics of $\alpha$-attractor in loop quantum cosmology. Phys. Rev. D 2018, 98, 043524. [CrossRef]

29. Shahalam, M. Preinflationary dynamics of power-law potential in loop quantum cosmology. Universe 2018, 4, 87. [CrossRef]

30. Sharma, M.; Shahalam, M.; Wu, Q.; Wang, A. Preinflationary dynamics in loop quantum cos-mology: Monodromy Potential. JCAP 2018, 11, 003. [CrossRef]

31. Almeida, C.R.; Fabris, J.C.; Sbisá, F.; Tavakoli, Y. Quantum cosmology with k-Essence theory. Phys. Math. Asp. Symmetries 2017. [CrossRef]

32. Tsamparlis, M.; Paliathanasis, A. Two dimensional dynamical systems which admit Lie and Noether symmetries. J. Phys. A Math. Theor. 2011, 44, 175202. [CrossRef] 